\title{
Simultaneous liver-kidney transplantation: A single-center experience in Korea
}

\author{
Minjae Kim ${ }^{1}$, Shin Hwang ${ }^{1}$, Chul-Soo Ahn ${ }^{1}$, Deok-Bog Moon ${ }^{1}$, Tae-Yong Ha ${ }^{1}$, \\ Gi-Won Song ${ }^{1}$, Dong-Hwan Jung ${ }^{1}$, Sung Shin ${ }^{2}$, Young Hoon $\mathrm{Kim}^{2}$, Hea-Seon $\mathrm{Ha}^{3}$, \\ Jung-Ja Hong ${ }^{3}$, In-Ok Kim ${ }^{3}$, Duck Jong Han ${ }^{2}$, and Sung-Gyu Lee ${ }^{1}$
}

\author{
${ }^{1}$ Division of Hepatobiliary Surgery and Liver Transplantation, Department of Surgery, ${ }^{2}$ Division of Kidney and \\ Pancreas Transplantation, Department of Surgery, ${ }^{3}$ Organ Transplantation Center, Asan Medical Center, University \\ of Ulsan College of Medicine, Seoul, Korea
}

\begin{abstract}
Backgrounds/Aims: Simultaneous liver and kidney transplantation (SLKT) has been established as the treatment of choice for patients with concurrent end-stage liver and end-stage kidney diseases. The objective of this study was to analyze the nationwide incidence of SLKT in Korea and the outcomes of SLKT in a high-volume transplant center. Methods: Databases of the Korean Network for Organ Sharing (KONOS) and Asan Medical Center from 2000 to 2019 were retrospectively reviewed to determine the incidence of SLKT. Results: During 20 years from 2000 to 2019, deceased donor SLKT was performed for 38 cases in the KONOS database. The proportion of deceased donor SLKT was $0.6 \%$ (20 of 3333 ) before adoption of MELD score, which was significantly increased to $1.2 \%$ (18 of 1524$)$ after the adoption of MELD score $(p=0.034)$. In our institution, there were 11 cases of SLKT (2 cases with deceased donors and 9 cases with living donors). SLKT accounted for $0.2 \%$ (11 of 6468) of total liver transplantation volume. During follow-up, five patients died due to hepatocellular carcinoma recurrence $(n=2)$, infection $(n=2)$, or unknown cause $(n=1)$. The 1-year and 10-year overall patient survival rates were $90.9 \%$ and $81.8 \%$, respectively. Conclusions: Results of this study revealed that the incidence of deceased donor SLKT was very low. An increase of such incidence is not anticipated unless the number of deceased donors is markedly increased. Currently, sequential living donor liver transplantation and kidney transplantation with deceased or living donors are mainstays of transplantation rather than SLKT in our institution. (Ann Hepatobiliary Pancreat Surg 2020;24:454-459)
\end{abstract}

Key Words: Hepatocellular carcinoma; Donor shortage; Chronic renal failure; Acute kidney injury; Living donor

\section{INTRODUCTION}

The first successful simultaneous liver and kidney transplantation (SLKT) was performed in $1983 .{ }^{1}$ Thereafter, SLKT has been established as the treatment of choice for patients with concurrent end-stage liver and end-stage kidney diseases. ${ }^{1,2}$ Currently, more than 400 SLKTs are performed annually in Europe and the United States. ${ }^{2-4}$ In contrast, SLKT is still a rare procedure in Korea. Only 38 cases of SLKT from deceased donors were registered to the Korean Network for Organ Sharing (KONOS) database from 2000 to 2019 . SLKT has been usually performed with different living liver and kidney donors in Korea. Since there are only a few case reports of SLKT from Korean transplant centers in the literature, ${ }^{5-8}$ information on SLKT in Korea is very limited. The objective of the present study was to analyze the nationwide incidence of SLKT and the outcomes of SLKT in a high-volume transplant center in Korea.

\section{MATERIALS AND METHODS}

\section{Study design}

Databases of the Korean Network for Organ Sharing (KONOS) and Asan Medical Center from January 2000 to December 2019 were retrospectively reviewed to determine the incidence of SLKT. The model for end-stage liver disease (MELD) score has been used for liver alloca-

Received: August 8, 2020; Revised: September 11, 2020; Accepted: September 13, 2020

Corresponding author: Shin Hwang

Department of Surgery, Asan Medical Center, University of Ulsan College of Medicine, 88 Olympic-ro 43-gil, Songpa-gu, Seoul 05505, Korea Tel: +82-2-3010-3930, Fax: +82-2-3010-6701, E-mail: shwang@amc.seoul.kr

Copyright (C) 2020 by The Korean Association of Hepato-Biliary-Pancreatic Surgery

This is an Open Access article distributed under the terms of the Creative Commons Attribution Non-Commercial License (http://creativecommons.org/ licenses/by-nc/4.0) which permits unrestricted non-commercial use, distribution, and reproduction in any medium, provided the original work is properly cited. Annals of Hepato-Biliary-Pancreatic Surgery • pISSN: 2508-5778 - eISSN: 2508-5859 


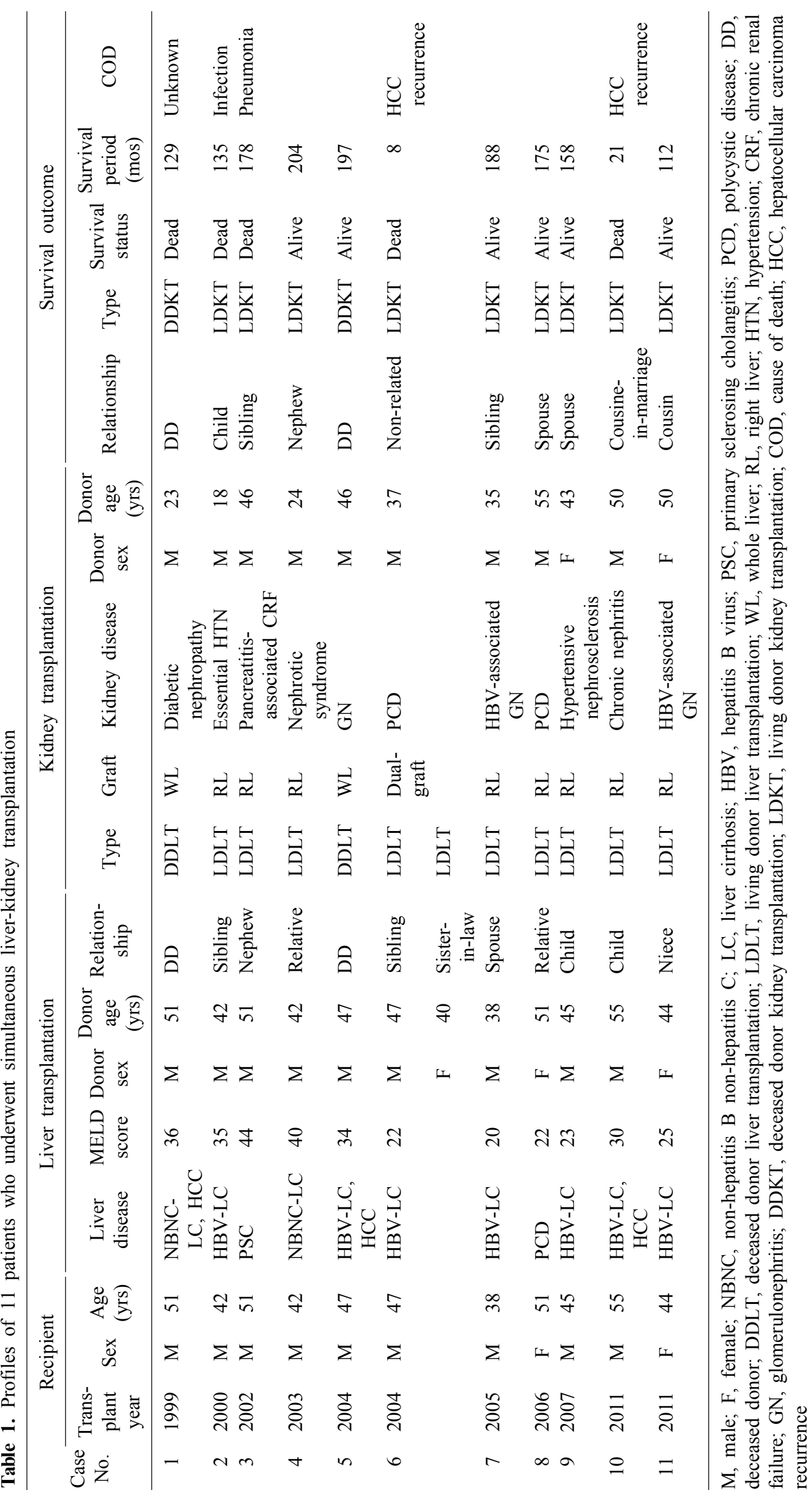


tion in Korea since 2016. The study protocol was approved by the Institutional Review Board (IRB) of our institution (IRB No. 2020-0857). The requirement for informed consent was waived by the IRB due to the retrospective nature of this study. This study was performed in accordance with ethical guidelines of the World Medical Association Declaration of Helsinki 2013.

\section{Statistical analysis}

Numerical data are presented as mean \pm standard deviation. Survival rates were estimated using the KaplanMeier method. Chi-square test was used for comparison of incidence variable. A $p$-value of less than 0.05 was considered statistically significant. All statistical analyses were performed using SPSS version 22 (IBM, New York, NY, USA).

\section{RESULTS}

\section{Incidence of SLKT in the KONOS database}

During the 20-year study period from January 2000 to December 2019, there were 38 cases of SLKT from deceased donors ( 1 case in 2004, 2007, and 2008 each; 4 cases in 2009; 3 cases in 2011, 2012, 2013, and 2014 each; 1 case in 2015; 4 cases in 2016; 7 cases in 2017; 4 cases in 2018; and 3 cases in 2019). During the same period, the number of total deceased donor liver transplantation (DDLT) and deceased donor kidney transplantation (DDKT) cases were 4857 (3333 cases before adoption of MELD score and 1524 cases after adoption of MELD score) and 10768 (7035 cases before adoption of MELD score and 3733 cases after adoption of MELD), respectively. The proportion of deceased donor SLKT was $0.6 \%$ (20 of 3333) before adoption of MELD score, which was significantly increased to $1.2 \%$ (18 of 1524) after the adoption of MELD score $(p=0.034)$.

\section{Profiles of SLKT in Asan Medical Center}

From January 2000 to December 2019, 6468 cases of LT (1056 cases of DDLT and 5412 cases of living donor liver transplantation [LDLT]) and 4818 cases of KT (1036 cases of DDKT and 3782 cases of living donor kidney transplantation [LDKT]) were performed in our institution. There were 11 cases of SLKT, 2 cases with deceased donors and 9 cases with living donors. SLKT ac- counted for $0.2 \%$ (11 of 6468) of total LT volume. Although $21.7 \%$ (1056 of 4857) of nationwide DDLT volume were performed in our institution during the study period, deceased donor SLKT accounted for only 5.3\% (2 of 38). There was no case of deceased donor SLKT after the introduction of MELD score.

Detailed profiles of these 11 cases of SLKT are summarized in Table 1. The mean MELD score was 30.1 18.2 . One patient (Case No. 8) with polycystic disease underwent bilateral nephrectomy due to recurrent infection at one year after SLKT. Three patients (Case No. 2, 3, and 9) showed acute rejection of kidney allograft which was managed with anti-rejection treatment. During a mean follow-up period of $137 \pm 67$ months, five patients died due to hepatocellular carcinoma $(\mathrm{HCC})$ recurrence $(n=2)$, infection $(n=2)$, and unknown cause $(n=1)$. The pretransplant extent of HCC was beyond Milan criteria in the two patients who showed posttransplant recurrence. The 1-year, 3-year, 5-year, 10-year, and 15-year overall patient survival rates were $90.9 \%, 81.8 \%, 81.8 \%, 81.8 \%$, and $46.0 \%$, respectively (Fig. 1).

\section{Profiles of KT before and after LT in Asan Medical Center}

Three patients had undergone KT first. After a mean period of 68 months (range, 20-104 months), they also underwent LT (all DDLTs). Thus, sequential KT-LT was performed in three patients.

In contrast, 34 patients had undergone LT first. After a mean period of 93 months (range, 1-234 months), they

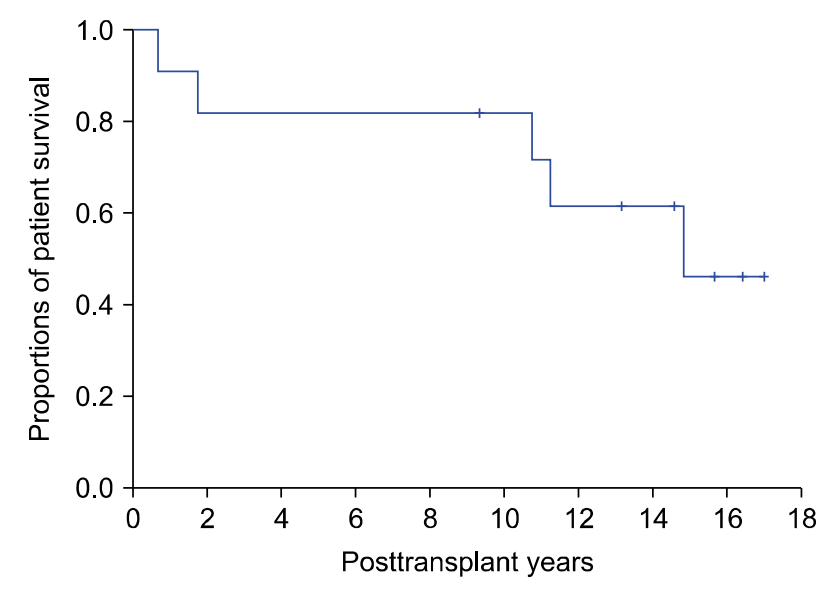

Fig. 1. Kaplan-Meier analysis of overall patient survival in 11 patients who underwent simultaneous liver-kidney transplantation. 
also underwent KT (DDKT in 11 and LDKT in 23). Thus, sequential LT-KT was performed in 34 patients. Currently, 51 patients are in the waiting list of DDKT. Eleven of them were enrolled before LT and 40 patients were enlisted after LT.

\section{DISCUSSION}

A considerable proportion of patients with both endstage liver and kidney diseases have undergone LT alone or SLKT worldwide. Recently, the proportion of SLKT among all LTs in the United States increased up to $9.9 \%$ in 2016. ${ }^{3,4}$ Meanwhile, because of profound deceased donor shortage in Korea, around $1 \%$ of LT candidates had undergone deceased donor SLKT in the current analysis of the KONOS database. In our institution, only $0.2 \%$ of total LT cases underwent SLKT with deceased or living donors. To the best of our knowledge, our Case No. 1 was the first case of SLKT in Korea. Considering that KT was performed in 3 patients before LT and 34 patients after LT, the sum of combined LT and KT was 48, accounting for $0.7 \%$ of total $\mathrm{LT}$ cases. In addition, 51 patients are currently enrolled in the waiting list of DDKT. Considering that there are many other patients showing slow but progressive deterioration of renal function after LT, the demand for KT in LT recipients would be higher than we calculated.

Information on SLKT in Korea is very limited. There are only a few case reports of SLKT from Korean transplant centers in the literature. ${ }^{5-8}$ A Korean single-center report on 3000 cases of $\mathrm{KT}$ has presented the performance of 16 cases of SLKT, ${ }^{9}$ however, detailed information on SLKT was not described. This was the primary reason that we carried out this study.

Renal disease has high impacts on LT candidates. Serum creatinine is included in the MELD scoring system because renal impairment is associated with a substantial increase in 30-day mortality, especially if an LT candidate requires dialysis. ${ }^{1,3}$ Renal impairment is a common occurrence in patients with liver disease. Some studies indicated that its prevalence is $15 \%-20 \%$ in LT candidates. ${ }^{10}$ The requirement to update MELD scores after the initial listing allows for allocation of a liver to those most in need.

Renal disease on LT recipients also has a high prog- nostic impact. The impact of kidney disease on LT candidates is not limited to pretransplant mortality. LT recipients who develop chronic kidney disease (CKD) following LT show significant increases of liver allograft failure and mortality compared to those who do not develop CKD after LT. Significant renal disease prior to LT is the most important risk factor for CKD after LT. Determining the cause of acute kidney injury in LT candidates can be challenging, which complicates the ability to assess potential renal recovery after LT. LT candidates on dialysis for more than 90 days with advanced CKD and prolonged acute kidney injury not from hepatorenal syndrome have a higher risk for significant renal impairment after LT. ${ }^{11}$

After the introduction of MELD criteria for allocation of LT in the United States, there was a more than $400 \%$ increase in SLKT, from $2.5 \%$ in 2001 to $9.9 \%$ in 2016. This change was driven primarily by poor outcomes of LT recipients with posttransplant kidney disease, an inability to predict which candidates were likely to develop post-LT CKD, and the lack of a mechanism to expedite $\mathrm{KT}$ in LT recipients who developed advanced CKD after $\mathrm{LT}^{11}$ The introduction of MELD score to the KONOS liver allocation system and an increase in the number of deceased donors raised the proportion of deceased donor SLKT from $0.6 \%$ before the adoption of MELD score to $1.2 \%$ after the adoption of MELD score in the present study. ${ }^{12-14}$ Although this increase was statistically significant, the proportion of SLKT was still very low compared to that in the United States. ${ }^{11,15}$

An analysis of United Network for Organ Sharing (UNOS)/Organ Procurement and Transplantation Network (OPTN) data has demonstrated a clear benefit of SLKT in LT candidates on dialysis for more than 2 months prior to LT and in those with a serum creatinine of greater than $2.5 \mathrm{mg} / \mathrm{dl}$, in which SLKT recipients show improved survival than those who receive LT alone. ${ }^{16}$ In addition, deceased donor SLKT can avoid immunological complexities of implanting two organs from different donors. Human leukocyte antigen tissue typing and compatibility have a much larger role in allograft survival in KT recipients. SLKT provides improved immunological outcomes in this setting. Based on these benefits of SLKT, a formal policy was enacted in 2017 in the United States with defined listing criteria for SLKT and priority for patients who had received LT and subsequently developed 
significant kidney disease after $\mathrm{LT}^{3}$

In the current KONOS allocation policy, multiple or two-organ transplantation does not have any priority for liver or kidney allocation. The primary background reasons of the increase in SLKT after introduction of MELD score in the KONOS allocation system might be the high MELD score per se from CKD in SLKT candidates and an increase of deceased donors at that time. However, considering that the daily cutoff MELD score for liver allocation is very high and the number of deceased donors is recently decreasing, it is difficult to expect that deceased donor SLKT would be continuously increasing afterwards. ${ }^{14}$ It is a dilemma to give a priority to SLKT candidates in the current Korean setting because separate allocation of kidney and liver organs can save two patients.

The majority of SLKTs in our institution were performed through a combination of LDLT and LDKT. It is usual to have two different donors for LDLT and LDKT. Although two-organ donation from a living donor has an immunologic benefit for LDKT, ${ }^{15,17,18}$ there are ethical and medical issues, especially issues regarding donor safety. One study supports such two-organ donation for pediatric patients. ${ }^{15}$ However, right liver donation for adult LT recipients might carry higher risk than left liver donation for pediatric patients.

SLKT with different liver and kidney living donors has different aspects from that with one deceased donor. In reality, half of adult LDLTs in Korea have been performed for patients with HCC. These patients carry risk of posttransplant HCC recurrence. ${ }^{19,20}$ In the present study, two of five patients died due to HCC recurrence. Sequential LDLT and KT with living or deceased donors would be beneficial to avoid risk of futile KT from early HCC recurrence after LT. We presume that it would be one of the underlying reasons to not prefer SLKT with living donors. As shown in results of this study, SLKT with living donors was not performed after 2011 in our institution. Sequential LT-KT has settled as a main modality of LT-KT combination.

This study has a notable limitation. It was a retrospective, single-center study with a small number of study patients. Further high-volume multicenter studies are needed to validate results of this study.

In conclusion, the results of this study revealed that the incidence of deceased donor SLKT was very low despite the introduction of MELD score. An increase of its incidence in the near future is not anticipated unless the number of deceased donors is markedly increased. Currently, sequential LDLT and KT with deceased or living donors is the main stay of transplantation rather than SLKT in our institution.

\section{ACKNOWLEDGEMENTS}

This study was supported by a Research Fund of Asan Medical Center Organ Transplantation Center.

\section{CONFLICT OF INTEREST}

None of the authors has any potential financial or other conflicts of interest relevant to this article.

\section{ORCID}

Minjae Kim: https://orcid.org/0000-0001-6743-0636

Shin Hwang: https://orcid.org/0000-0002-9045-2531

Chul-Soo Ahn: https://orcid.org/0000-0002-3844-3646

Deok-Bog Moon: https://orcid.org/0000-0002-8209-3540

Tae-Yong Ha: https://orcid.org/0000-0001-9932-0212

Gi-Won Song: https://orcid.org/0000-0002-4235-0434

Dong-Hwan Jung: https://orcid.org/0000-0001-5984-023X

Sung Shin: https://orcid.org/0000-0001-7318-4208

Young Hoon Kim: https://orcid.org/0000-0003-3840-8426

Hea-Seon Ha: https://orcid.org/0000-0003-1923-7481

Jung-Ja Hong: https://orcid.org/0000-0002-8219-3170

In-Ok Kim: https://orcid.org/0000-0001-8615-9191

Duck Jong Han: https://orcid.org/0000-0002-0990-6824

Sung-Gyu Lee: https://orcid.org/0000-0001-9161-3491

\section{AUTHOR CONTRIBUTIONS}

Conceptualization: SH. Data curation: CSA, DBM, TYH, GWS, DHJ, SS, YHK. Methodology: DJH, HSH, JJH, IOK, SGL. Visualization: SH. Writing - original draft: MK, SH. Writing - review \& editing: SH.

\section{REFERENCES}

1. Margreiter R, Kramar R, Huber C, Steiner E, Niederwieser D, 
Judmaier G, et al. Combined liver and kidney transplantation. Lancet 1984;1:1077-1078.

2. Formica RN, Aeder M, Boyle G, Kucheryavaya A, Stewart D, Hirose R, et al. Simultaneous liver-kidney allocation policy: a proposal to optimize appropriate utilization of scarce resources. Am J Transplant 2016;16:758-766.

3. Lum EL, Cárdenas A, Martin P, Bunnapradist S. Current status of simultaneous liver-kidney transplantation in the United States. Liver Transpl 2019;25:797-806.

4. Miles CD, Westphal S, Liapakis A, Formica R. Simultaneous liver-kidney transplantation: impact on liver transplant patients and the kidney transplant waiting list. Curr Transplant Rep 2018; $5: 1-6$

5. Wi W, Hahm TS, Kim GS. A case series on simultaneous liver and kidney transplantation: do we need intraoperative renal replacement therapy? Korean J Anesthesiol 2017;70:467-476.

6. Nah YW, Nam CW, Suh JH, Cha HJ, Kim GY, Park SJ, et al. Isolated acute cellular rejection of the liver after simultaneous liver and kidney transplantation: a case report. Transplant Proc 2008;40:2832-2834.

7. Chae MS, Kim Y, Oh SA, Jeon Y, Choi HJ, Kim YH, et al. Intraoperative management of a patient with impaired cardiac function undergoing simultaneous $\mathrm{ABO}$-compatible liver and ABO-incompatible kidney transplant from 2 living donors: a case report. Transplant Proc 2018;50:3988-3994.

8. Kim K, Jeong DW, Lee YH, Kim YG, Moon JY, Jeong KH, et al. Everolimus-induced systemic serositis after simultaneous liver and kidney transplantation: a case report. Transplant Proc 2017;49:181-184.

9. Go J, Ko K, Jun D, Kwon SK, Han S, Kim YH, et al. A half-century 3000 cases of kidney transplant experiences in a single hospital: the longest registry in Korea. Transplant Proc 2019; 51:2559-2567.

10. Gleisner AL, Jung H, Lentine KL, Tuttle-Newhall J. Renal dysfunction in liver transplant candidates: evaluation, classification and management in contemporary practice. J Nephrol Ther 2012; Suppl 4(SI Kidney Transplantation):006.

11. O'Riordan A, Wong V, McCormick PA, Hegarty JE, Watson AJ.
Chronic kidney disease post-liver transplantation. Nephrol Dial Transplant 2006;21:2630-2636.

12. Lee J, Lee JG, Jung I, Joo DJ, Kim SI, Kim MS; Advisory Committee on Improving Liver Allocation. Development of a Korean liver allocation system using model for end stage liver disease scores: a nationwide, multicenter study. Sci Rep 2019;9: 7495.

13. Min SI, Ahn C, Han DJ, Kim SI, Chung SY, Lee SK, et al. To achieve national self-sufficiency: recent progresses in deceased donation in Korea. Transplantation 2015;99:765-770.

14. Ha HS, Hong JJ, Kim IO, Lee SR, Lee AY, Ha TY, et al. Deceased donor liver transplantation under the Korean model for end-stage liver disease score-based liver allocation system: 2-year allocation results at a high-volume transplantation center. Korean J Transplant 2019;33:112-117.

15. Gautier S, Monakhov A, Tsiroulnikova O, Voskanov M, Miloserdov I, Dzhanbekov T, et al. Deceased vs living donor grafts for pediatric simultaneous liver-kidney transplantation: a single-center experience. J Clin Lab Anal 2020;34:e23219.

16. Fong TL, Khemichian S, Shah T, Hutchinson IV, Cho YW. Combined liver-kidney transplantation is preferable to liver transplant alone for cirrhotic patients with renal failure. Transplantation 2012;94:411-416.

17. Kitajima K, Ogawa Y, Miki K, Kai K, Sannomiya A, Iwadoh $\mathrm{K}$, et al. Longterm renal allograft survival after sequential liver-kidney transplantation from a single living donor. Liver Transpl 2017;23:315-323.

18. Marujo WC, Barros MF, Cury RA, Pacheco-Silva A, Sette H Jr. Successful combined kidney-liver right lobe transplant from a living donor. Lancet 1999;353:641.

19. Lee SG, Moon DB, Hwang S, Ahn CS, Kim KH, Song GW, et al. Liver transplantation in Korea: past, present, and future. Transplant Proc 2015;47:705-708.

20. Yoon YI, Song GW, Lee SG, Hwang S, Kim KH, Kim SH, et al. Outcome of ABO-incompatible adult living-donor liver transplantation for patients with hepatocellular carcinoma. J Hepatol 2018;68:1153-1162. 